\title{
ARTICLE
}

\section{External fixation is more suitable for intra-articular fractures of the distal radius in elderly patients}

\author{
Chuang $\mathrm{Ma}^{1, *}$, Qiang Deng ${ }^{1, *}$, Hongwei $\mathrm{Pu}^{2}$, Xinchun Cheng ${ }^{3}$, Yuhua $\mathrm{Kan}^{4}$, Jing Yang ${ }^{5}$, \\ Aihemaitijiang Yusufu ${ }^{1}$ and $\mathrm{Li} \mathrm{Cao}^{1}$
}

The purpose of this study was to compare the functional outcomes, psychological impact, and complication rates associated with external fixation and volar or dorsal plating in relation to the functional parameters following treatment of intra-articular fractures of the distal radius (IFDR) in patients older than 65 years. We hypothesized that using volar or dorsal plating would improve functional outcomes, but that it would be associated with more complications and equivalent functional outcomes when compared with the external fixation group. A total of 123 consecutive patients suffering from IFDR were recruited into the study. The patients were measured for clinical, radiological, and psychosocial functioning outcomes and were followed up after 1 week and 3, 6 and 12 months. After 3 months, the plating group had better pronation $(P=0.001)$, supination, $(P=0.047)$ and extension $(P=0.043)$ scores. These differences were somewhat attenuated by 6 months and disappeared at 1 year. The plating group had a greater occurrence of wound infection $(P=0.043)$, tendonitis, $(P=0.024)$ and additional surgery compared with the external fixation group. The only TNO-AZL Adult Quality of Life scores in the plating group that were lower than those in the external fixation group were in the "gross motor" category (walking upstairs, bending over, walking 500 yards; $P=0.023$ ). Internal fixation was more advantageous than external fixation in the early rehabilitation period; after 1 year the outcomes were similar. The plating group showed significantly higher levels of wound infection and tendonitis and had a greater need for additional surgeries.

Bone Research (2016) 4, 16017; doi:10.1038/boneres.2016.17; published online: 21 June 2016

\section{INTRODUCTION}

Intra-articular fractures of the distal radius (IFDR) represent high energy, complex, unstable injuries, and account for approximately one-sixth of the fractures observed in emergency rooms. ${ }^{1-2}$ The risk of a poor outcome after IFDR increases with malunion and joint stiffness, and operations are often required to maintain a satisfactory anatomical position. ${ }^{3}$ Over the past 30 years, the surgical treatment of distal radius fractures has shifted from the use of cast immobilization to numerous surgical options, such as the use of external fixation and locking plates. ${ }^{4-5}$ Although many reports on the functional outcomes, psychological impacts and complication rates have been reported for the use of locked plating fixation or external fixation around the wrist, ${ }^{6-7}$ little has been written about these issues in elder patients. ${ }^{8}$ Chung et al. ${ }^{9}$ reported complication rates after volar-locked plating of distal radius fractures; however, they included both young adults and adults older than 60 years in their analysis and did not focus on the elderly population.

Procedures for treating IFDR can be psychologically stressful because of their long duration and the frequency of complications, including soft tissue problems and restricted joint motion. Thus, the procedure used for

\footnotetext{
'Department of Orthopedics Center, First Affiliated Hospital of Xinjiang Medical University, Urumai, China; ${ }^{2}$ Department of Science and Research Education Center, First Affiliated Hospital of Xinjiang Medical University, Urumqi, China; ${ }^{3}$ Carders Health Care NO. 4 Department of Xinjiang Uygur Autonomous Region People's Hospital, Urumai, China; ${ }^{4}$ Department of Orthopedics of the Central Hospital of Karamay, Karamay, China and ${ }^{5}$ Department of Orthopedics Center, Fifth Affiliated Hospital of Xinjiang Medical University, Urumqi, China

*These authors contributed equally to this work.

Correspondence: Aihemaitijiang Yusufu (aihemaitijiang@163.com) and Li Cao (1978589785@qq.com)

Received: 26 November 2015; Revised: 18 May 2016; Accepted: 19 May 2016
} 
treating IFDR can have a strong psychological impact on the patient.

There are obvious benefits and disadvantages of these two surgical techniques and the associated post-operative rehabilitation protocols. Some authors have previously compared external fixation with internal fixation, but there is insufficient evidence regarding which procedure has the best outcome in elderly patients. ${ }^{10-13}$

The primary purpose of this study was to compare the functional outcomes, psychological impacts, and complication rates in elderly patients whose IFDR was treated by either external or internal fixation.

\section{MATERIALS AND METHODS}

All studies involving human participants were approved by the First Affiliated Hospital of Xinjiang Medical University. Before operation, written informed consent was provided by participants for their clinical records to be used in this study, and the consent process was carried out by the treating surgeon. This case-control study excluded patients with open fractures, previous surgery involving the distal radius, concurrent injury involving the ipsilateral upper limb, and wrist pain predating this injury. Two hundred consecutive elderly patients suffering from IFDR between June 2009 and May 2015 were considered for the study. Of these, 123 patients met the inclusion criteria of a fracture of the distal radius requiring operative repair amenable to either open reduction and internal fixation or external fixation. The Institution Review Board approved the study, and the patients provided informed consent. Baseline clinical details, including the mechanisms of the fractures, and wrist, shoulder, and hand functional scores, were obtained. For each patient, a thorough and detailed patient history was obtained and a detailed physical examination was performed, with attention to neurovascular injury and deficits. The fractures were classified according to the system of the Orthopaedic Trauma Association (AO/OTA). ${ }^{14}$

The two groups were compared with respect to functional, clinical, and radiographic outcomes. Standard radiographs were obtained at presentation, including anteroposterior and lateral views. Radiographs of all displaced fractures were obtained. Measurements of radial inclination, height, tilt, and ulnar variance were made on post-reduction radiographs (Table 1).

There were no differences between the two groups in regard to age, gender, pattern of fracture, hand dominance, or number of pre-existing medical conditions (cardiovascular, endocrine, pulmonary, or kidney disease; Table 2). The patients were randomized into two groups according to whether they underwent internal fixation or external fixation. A number for each patient was placed in a sealed envelope by a blinded assistant and given to the
Table 1. Mean radiological measurements in the two groups

\begin{tabular}{|c|c|c|c|}
\hline Variables & External fixation & Plating & P-value \\
\hline \multicolumn{4}{|l|}{ Pre-reduction } \\
\hline Volar tilt/ ${ }^{\circ}$ & $-14.32( \pm 2.33)$ & $-14.82( \pm 2.56)$ & 0.52 \\
\hline Radial inclination $/^{\circ}$ & $13.63( \pm 1.3)$ & $13.89( \pm 1.56)$ & 0.67 \\
\hline Radial length/mm & $6.83( \pm 2.14)$ & $6.95( \pm 1.54)$ & 0.89 \\
\hline \multicolumn{4}{|l|}{ Post-reduction } \\
\hline Volar tilt/ ${ }^{\circ}$ & $2.21( \pm 1.12)$ & $2.31( \pm 1.52)$ & 0.54 \\
\hline Radial inclination $/^{\circ}$ & $17.76( \pm 2.32)$ & $17.56( \pm 2.15)$ & 0.34 \\
\hline radial length/mm & $8.21( \pm 2.33)$ & $7.76( \pm 2.46)$ & 0.32 \\
\hline \multicolumn{4}{|l|}{6 months pos operation } \\
\hline Volar tilt/ ${ }^{\circ}$ & $3.45( \pm 1.87)$ & $3.21( \pm 1.77)$ & 0.12 \\
\hline Radial inclination $/{ }^{\circ}$ & $19.25( \pm 3.97)$ & $20.24( \pm 2.89)$ & 0.56 \\
\hline Radial length/mm & $8.87( \pm 3.65)$ & $8.90( \pm 3.55)$ & 0.59 \\
\hline \multicolumn{4}{|c|}{12 months post operation } \\
\hline Volar tilt/ ${ }^{\circ}$ & $3.56( \pm 1.21)$ & $3.31( \pm 1.23)$ & 0.87 \\
\hline Radial inclination $/{ }^{\circ}$ & $19.32( \pm 3.76)$ & $20.12( \pm 3.32)$ & 0.8 \\
\hline Radial length/mm & $9.13( \pm 2.13)$ & $9.34( \pm 2.89)$ & 0.78 \\
\hline
\end{tabular}

Table 2. Clinical details of the patients in the two groups

\begin{tabular}{|c|c|c|c|}
\hline Characteristics & External fixation & Plating & P-value \\
\hline Age & $67.2(19-75)$ & $68.7(19-81)$ & 0.326 \\
\hline Gender & & & 0.546 \\
\hline Male & 28 & 30 & \\
\hline Female & 30 & 35 & \\
\hline Hand dominance & & & 0.446 \\
\hline Right & 26 & 41 & \\
\hline Left & 32 & 24 & \\
\hline Medial nerve injury & & & 0.215 \\
\hline Yes & 9 & 6 & \\
\hline No & 49 & 59 & \\
\hline Associated with ulnar fracture & & & 0.464 \\
\hline Yes & 5 & 7 & \\
\hline No & 53 & 58 & \\
\hline \multicolumn{4}{|l|}{ Co-morbidities } \\
\hline Cardiovascular disease & & & 0.269 \\
\hline Yes & 2 & 5 & \\
\hline No & 56 & 60 & \\
\hline Endocrine disease & & & 0.302 \\
\hline Yes & 10 & 8 & \\
\hline No & 48 & 57 & \\
\hline Pulmonary disease & & & 0.554 \\
\hline Yes & 2 & 3 & \\
\hline No & 56 & 62 & \\
\hline Kidney disease & & & 0.723 \\
\hline Yes & 1 & 1 & \\
\hline No & 57 & 64 & \\
\hline
\end{tabular}

treating surgeon. The envelopes were opened on the day of surgery, immediately before the operation. Fifty-eight patients were randomly assigned to the external fixation treatment group and 65 to the internal fixation treatment group. The criteria used to identify unacceptable reductions were a radial height shortening of $>5 \mathrm{~mm}$, radial inclination on a postero-anterior film $<15^{\circ}$, intra-articular step off $>2 \mathrm{~mm}$, dorsal tilt $>15^{\circ}$ and volar tilt $>20^{\circ} .{ }^{15}$ 

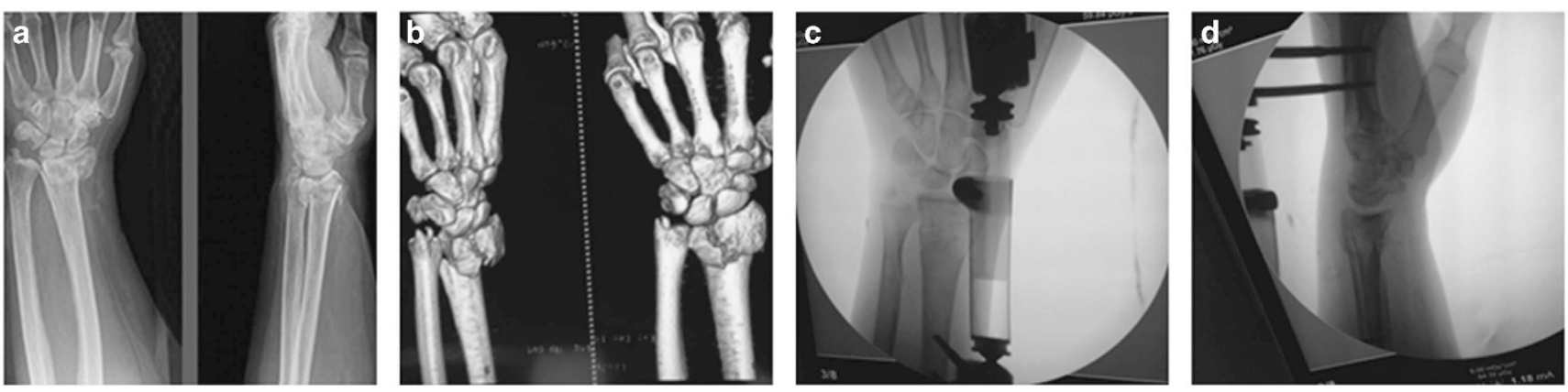

Figure 1. Radiographs of a 68-year-old man with a displaced intra-articular fracture of the distal radius that was treated by external fixation. (a and b) Anteroposterior and lateral radiograph before operation; (c and d) Anteroposterior and lateral radiograph during operation.
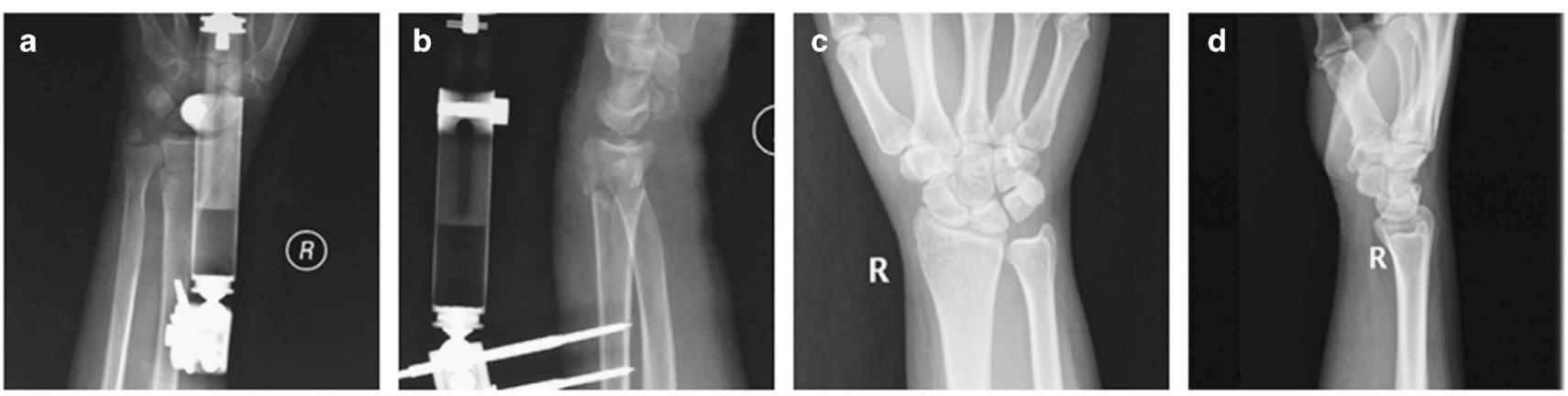

Figure 2. Radiographs of the distal radius with a comminuted intra-articular fracture after external fixation. (a and b) Anteroposterior and lateral radiographs after one month, (c and $\mathbf{d}$ ) Anteroposterior and lateral radiographs after one year.

Operative technique

All operations were performed by one of four senior orthopedic surgeons, who had been in practice for 6-10 years and had extensive experience performing external fixation or locked plating treatments. A full-time physiotherapist performed the pre- and post-operation clinical examinations. All the operations were performed under brachial plexus anesthesia or general anesthesia.

\section{External fixation}

The external fixation group underwent closed reduction or open reduction with the placement of two pins in the distal third of the radius and two pins in the base of the second metacarpal in an open surgical manner. If acceptable alignment was achieved, percutaneous Kirschner wires (K-wires) were placed to hold the reduction (Figure 1). Then, an external fixator (Orthofix or Stryker, AO, NJ, USA, depending on the surgeon's preference) was used to fix the fracture (Figure 2). If acceptable alignment could not be achieved, one incision was made to perform the anatomical reduction, and the fractured fragments were fixed with K-wires. A C-arm was used to check for alignment, reduction and screw placement (Figure 1).
Open reduction and internal fixation

A volar, dorsal, or volar/dorsal approach was used, and the plates used were $3.5 \mathrm{~mm}$ AO T or oblique plates. The fracture was reduced, and two pins were applied to achieve temporary fixation (Figure 3). Each fragment was identified, elevated, and reduced. Because the palmar surface of the distal radius is originally flat, application of a flat plate onto this surface was a good way to correct malrotations of the fractures in many cases (Figure 4). A $C$-arm was used to check for alignment, reduction and screw placement (Figure 3).

\section{Follow-up}

Clinical follow-up was conducted at 2 and 6 weeks, and 3 , 6 , and 12 months postoperatively. After 1 week, patients treated via external fixation began finger exercises. Patients were seen every month for radiological followups. A removable plaster cast was placed on patients in the plating group to allow free movement of their wrists and fingers.

After 3 weeks, the K-wires were removed in the outpatient clinic without anesthesia. External fixators were removed after 6 weeks. All patients had passive finger, 

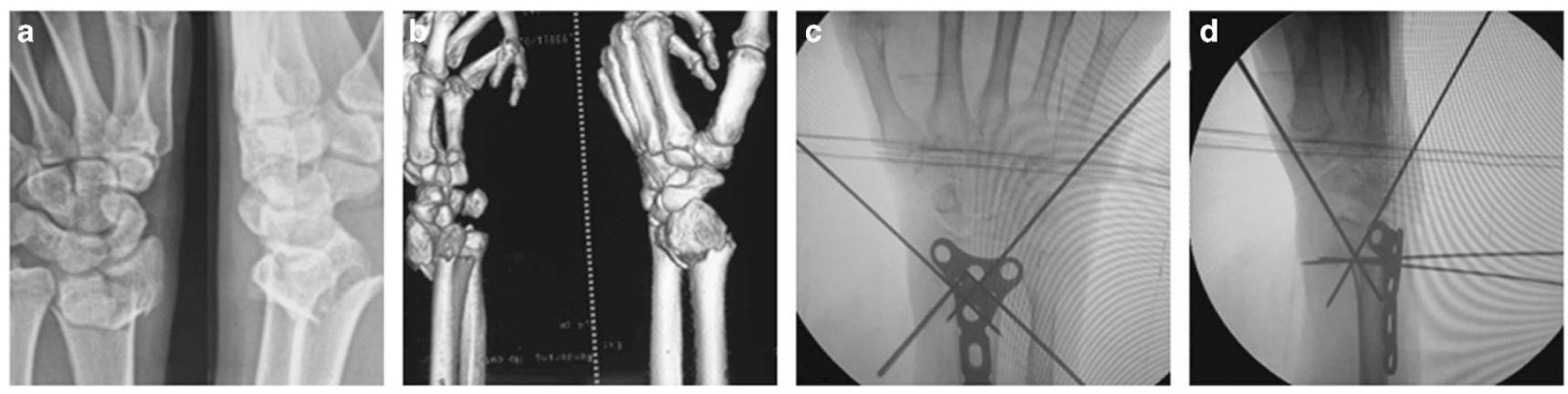

Figure 3. Radiographs of 73-year-old man with a comminuted intra-articular fracture of the distal radius that required treatment via Dorsal locked plating. (a and $\mathbf{b}$ ) Anteroposterior and lateral radiographs before operation; (c and $\mathbf{d}$ ) Anteroposterior and lateral radiographs during operation.
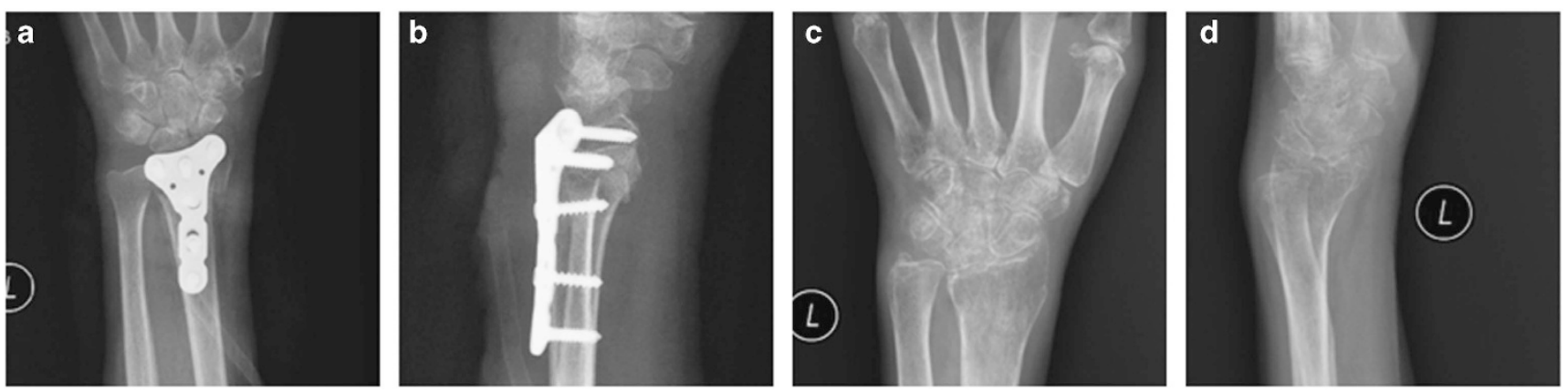

Figure 4. Radiographs of the man with a comminuted intra-articular fracture of the distal radius after treated by Dorsal locked plating. (a and $\mathbf{b}$ ) Anteroposterior and lateral radiographs after one month, (c and d) Anteroposterior and lateral radiographs after one year.

wrist, and forearm movement. Patients underwent formal physiotherapy emphasizing activities that could prevent join stiffness and tendon adhesions. A goniometer was used to measure the range of movement of the wrist and fingers.

Two validated Swedish versions of the DASH score ${ }^{14,16-17}$ questionnaire were used to assess patient-rated functional results. Both questionnaires yielded a score from 0 to 100; higher scores represented greater disability. The questionnaires were completed at baseline and after 3, 6, and 12 months. Scores are presented here with the baseline scores subtracted.

After 3,6 , and 12 months, grip strength and range of movement were assessed by a physiotherapist as objective function tests. The uninjured wrists were assessed as controls. Grip strength and range of movement are expressed as percentages of those of the uninjured hand and wrist. Grip strength was adjusted by $10 \%$ for the nondominant side. Pain was rated on a 10-point visual analog scale (0, no pain; 10 , severe pain).

At each post-operative visit, radiological assessment was performed using standard anteroposterior and lateral radiographs to evaluate the union of the fracture, loss of reduction, and development of arthritis. Radial inclination, height, and tilt were measured on each radiograph (Table 1). The presence of arthritic changes was noted when observing radiographs from the postoperative visits at 6 and 12 months. All radiographs were read by a senior radiologist who had been in practice for 10 years and had extensive experience reading radiographs.

\section{Perceived competence}

Two measures were used to investigate perceived competence. First, a Dutch version of the Self-Perception Profile was used to measure perceived competence for patients ${ }^{18}$ in six categories: scholastic competence, social acceptance, athletic competence, physical appearance, behavioral conduct, and global self-worth. Scores were expressed on a scale from 0 to 100. Second, a Dutch version of the Self-Perception Profile for Adolescents (CBSA) was used to measure self-esteem in patients.

\section{Health-related quality of life}

The TNO-AZL Adult Quality of Life questionnaire (TAAQOL) was used to investigate health-related quality of life (HRQOL) at follow-up. ${ }^{19}$ The questionnaire contained 45 questions, divided into 12 categories. Each category consisted of 2-4 questions pertaining to gross motor 
Table 3. Comparison of scores at the 3-, 6-, and 12-month follow-up in the two treatment groups

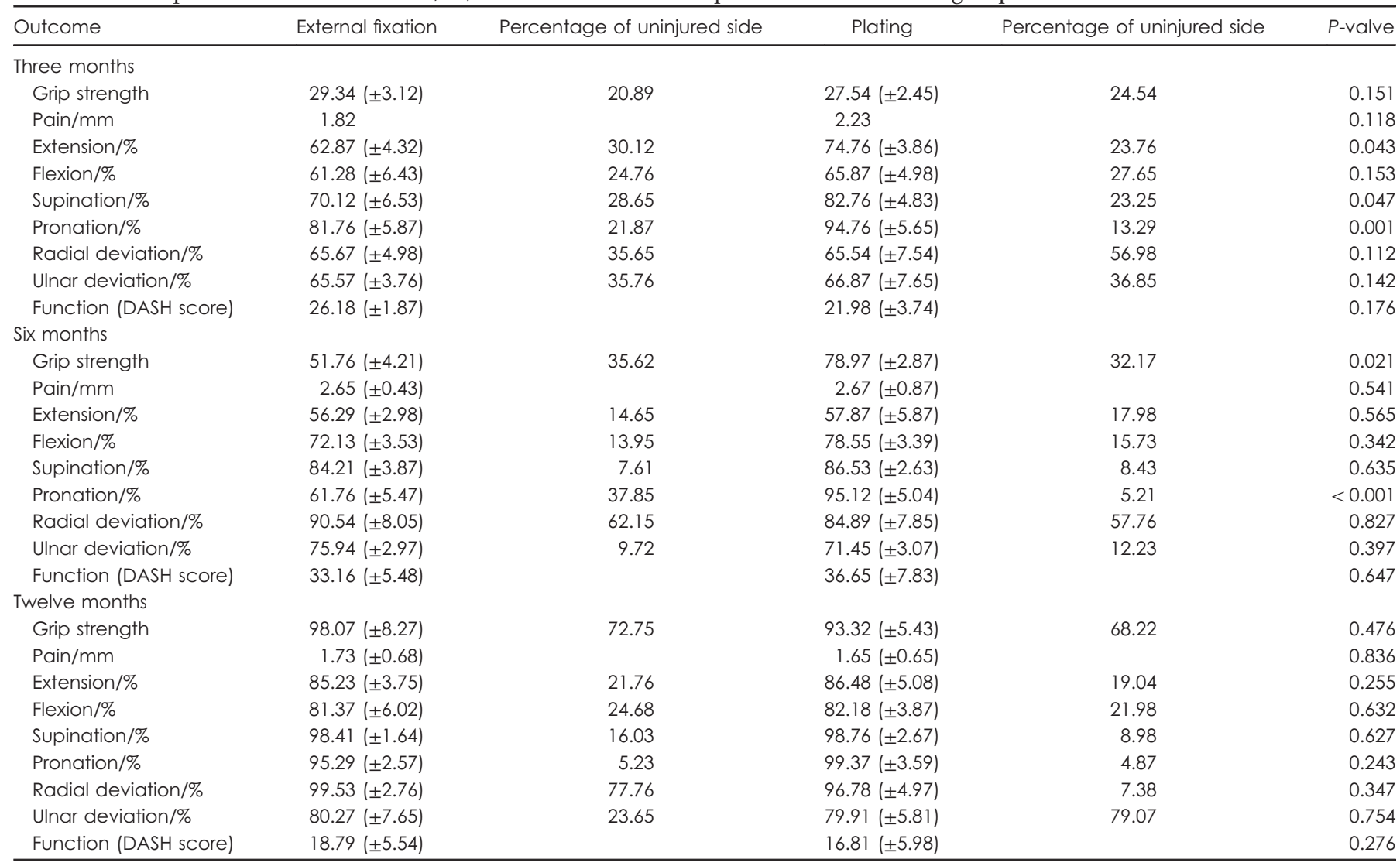

functioning (4), fine motor functioning (4), pain (4), sleep (4), cognitive functioning (4), social functioning (4), daily activities (4), sexual activity (2), vitality (4), happiness (4), depressive feelings (4), and aggressiveness (3). The total score came from a simple subscale values, and higher scores demonstrated a higher quality of life.

Statistics

The groups were compared using a $\chi^{2}$-test for gender, hand dominance, fracture classification, income level, and co-morbidities, $t$-tests were used to assess differences in range of movement and radiological measurements, and a linear regression was used to analyze the follow-up DASH scores while controlling for baseline scores. The level of statistical significance was set at $P<0.05$ for each test. All statistical analyses were performed using SPSS version 17.0 software (SPSS, Chicago, IL, USA).

\section{RESULTS}

The groups had equal distribution in age, gender, injured side, type of work, classification of fracture, radiographic findings, and type of injury (Table 2). ALL patients had intraarticular fractures, in either the radiocarpal joint, the distal radioulnar joint, or both. The operations were performed a mean of 4.6 (1-12) days after the injury occurred. In total, 58 patients received external fixation and 65 received plating. All patients underwent follow-up for at least 1 year.

There were no open fractures in the external fixation group or the plating group. At presentation, nine patients in the external fixation group and six patients in the plating group had symptoms of carpal tunnel syndrome. The mean length of the operative procedure was $85.8 \mathrm{~min}$ (52160) for the plating group and $83.6 \mathrm{~min}(50-150)$ for the external fixation group. Between the surgery and the subsequent 6 months, the patients in both groups attended an equal number of physiotherapy sessions.

There were no differences in the mean pain scores obtained at each interval for each group. The mean DASH scores at each follow-up point were similar for each group and were not significantly different. At each successive assessment, the mean range of wrist movement improved for each group (Table 3). There were obvious differences between the groups in pronation $(P<0.001)$, supination $(P=0.047)$, and extension $(P=0.043)$, which reached statistical significance after 3 months. These differences were somewhat attenuated by 6 months and disappeared after 1 year. For each group, differences in the mean range of grip strength did not reach statistical significance at any point during the follow-up period. 
Complication rates were not similar between the treatment methods (Table 4). There were obvious differences in rates of wound infection $(P=0.043)$, pin-track infection $(P=0.000)$, tendonitis $(P=0.024)$, and need for further surgery, which reached statistical significance. One patient in the external fixation group and six in the plating group acquired deep wound infections. One patient in the external fixation group and seven in the plating group required further surgery because of these infections or other complications. In the plating group, one patient underwent capsulectomy and tenolysis for post-operative stiffness and one had a tendon transfer to treat rupture of the extensor pollicis longus. Ten patients had their hardware removed and required an incision and drainage with the administration of antibiotics for the treatment of a post-operative wound infection. Three patients had their hardware removed because of the symptomatic prominence of the plate and screws; two developed nonunion and required further fixation with an external fixator and bone graft, with subsequent union. For multivariate analysis, the variables that were significant in the univariate analysis were subsequently analyzed using a Cox proportional hazard model. The factor significantly

Table 4. Details of the complications in both groups

\begin{tabular}{lccc}
\hline Details of the complications & External fixation & Plating & $P$-value \\
\hline Post-operative nerve deficit & 1 & 3 & 0.367 \\
Wound infection & 1 & 6 & 0.043 \\
Pin-track infection & 8 & 0 & 0.000 \\
Painful-retained hardware & 0 & 1 & 0.343 \\
Tendon rupture & 1 & 2 & 0.627 \\
Tendonitis & 1 & 8 & 0.024 \\
Nonunion & 1 & 2 & 0.627 \\
Further surgery & 1 & 7 & 0.042 \\
\hline
\end{tabular}

Table 5. Univariate analysis of risk factors relative to cure rate (logistic regression analysis)

\begin{tabular}{lcc}
\hline Clinicopathological variables & Wals & $P$-value \\
\hline Gender & 1.376 & 0.775 \\
Age & 1.545 & 0.856 \\
Bone loss & 2.653 & 0.432 \\
AO classification of fracture & 3.446 & 0.223 \\
Wound infection & 3.216 & 0.043 \\
\hline
\end{tabular}

AO, Orthopaedic Trauma Association. related to patient cure rates was wound infection (Table 5 and Table 6).

\section{Perceived competence}

Comparative data for perceived competence in the external fixation group and the plating group were available for 123 patients who completed the CBSA assessments after the procedure (Table 7). Most of the perceived competence scores in the external fixation group fell within the same average range as those from the plating group, except those for "athletic competence" and "close friendship", but there were no statistically significant differences in perceived competence between the two groups.

Health-related quality of life

Data comparing HRQOL in the external fixation group with that in the plating group were available for 123 patients who completed the TAAQoL assessment after the procedure (Table 8). The TAAQoL scores in the plating group were lower than those in the external fixation group in the "gross motor" categories (walking upstairs, bending over, and walking 500 yards; $P=0.023$; Table 8$)$. There were no statistically significant differences in perceived competence in the other categories (Table 5 and Table 8).

\section{DISCUSSION}

Improved results were found with regard to range of movement in the patients undergoing plating for treatment of IFDR in the early rehabilitation period compared with those who had external fixation, but after 1 year the outcomes were similar.

Table 7. Mean scores and standard deviation of perceived competence (ranged from 0 to 100) in the two treated groups. Higher scores indicate higher perceived competence.

\begin{tabular}{lccc}
\hline Scale & External fixation & Plating & $P$-value \\
\hline Scholastic competence & $54 \pm 6$ & $64 \pm 7$ & 0.342 \\
Social acceptance & $38 \pm 5$ & $61 \pm 4$ & 0.263 \\
Athletic competence & $41 \pm 3$ & $42 \pm 5$ & 0.567 \\
Physical appearance & $51 \pm 6$ & $56 \pm 6$ & 0.846 \\
Behavioral conduct & $53 \pm 7$ & $55 \pm 3$ & 0.913 \\
Close friendship & $57 \pm 2$ & $51 \pm 3$ & 0.646 \\
Global self-worth & $56 \pm 7$ & $61 \pm 4$ & 0.265 \\
Overall & $53 \pm 4$ & $55 \pm 5$ & 0.734 \\
\hline
\end{tabular}

Table 6. Multivariate analysis of risk factors relative to cure rate (logistic regression analysis)

\begin{tabular}{lccc}
\hline Independent factor & P-value & Regression coefficient & 95\% confidence interval \\
\hline Bone loss & 0.232 & 0.763 & $0.412(0.147-0.837)$ \\
AO classification of fracture & 0.225 & 0.853 & $0.687(0.267-0.978)$ \\
Wound infection & 0.027 & 0.624 & $0.432(0.213-0.746)$ \\
\hline
\end{tabular}

AO, Orthopaedic Trauma Association. 
Table 8. Mean HRQoL scores (TAAQoL; ranged from 0 to 100) in the two treated groups

\begin{tabular}{lccc}
\hline Scale & External fixation & Plating & $P$-value \\
\hline Gross motor & $91 \pm 9$ & $62 \pm 7$ & 0.026 \\
Fine motor & $90 \pm 8$ & $87 \pm 6$ & 0.767 \\
Cognitive function & $98 \pm 10$ & $96 \pm 4$ & 0.686 \\
Sleeping & $57 \pm 5$ & $88 \pm 7$ & 0.045 \\
Pain & $70 \pm 6$ & $72 \pm 7$ & 0.445 \\
Social function & $90 \pm 6$ & $94 \pm 8$ & 0.654 \\
Daily activities & $82 \pm 4$ & $82 \pm 5$ & 0.727 \\
Sexuality & $95 \pm 7$ & $93 \pm 7$ & 0.887 \\
Vitality & $66 \pm 5$ & $65 \pm 6$ & 0.748 \\
Happiness & $75 \pm 4$ & $73 \pm 8$ & 0.762 \\
Depressive feelings & $83 \pm 8$ & $97 \pm 9$ & 0.253 \\
Aggressiveness & $85 \pm 7$ & $82 \pm 5$ & 0.524 \\
\hline
\end{tabular}

HRQoL, health-related quality of life; TAAQoL, TNO-AZL Adult Quality of Life Higher scores indicate better quality of life.

Egol et al. ${ }^{20}$ compared volar-locked plating and bridging external fixation with the use of supplementary K-wires in 280 patients and found an improved range of movement early after volar plating, but after 1 year the range of movement between the groups was similar, as were the results for grip strength and DASH scores at all time points. Marcheix et $\mathrm{al}^{21}$ analyzed results from 103 randomized patients with unstable extra- and intra-articular fractures treated with volar-locked plating or "mixed pinning". The plated patients had better objective functional results and reported better DASH scores after 3 and 6 months. The 1 -year results were not reported. These results are in accordance with our results, although we found better grip strength in the plating group after 6 months. Patients undergoing plate fixation had more activity than did the patients undergoing external fixation in the early stages, which may explain this difference. Our patients undergoing external fixation had somewhat better radiographic results than the patients in a study by Wilcke et $a .^{22}$ This could be explained by the fact that some of our patients underwent open reduction and percutaneous $\mathrm{K}$-wires were placed to hold the reduction. We found that there was not a direct relationship among the differences in grip strength and DASH score and the radiographic results because the results show that even a potentially suboptimal external fixation method produced outcomes similar to those of plating after 1 year. The minor improvements in the range of movement seen early did not lead to a better functional outcome at any stage. ${ }^{20}$ Compared with the external fixation group, the plating group demonstrated improved wrist movement early on, but by 1 year most of these differences were not present.

Obtaining and maintaining an acceptable reduction and allowing restoration of function are the goals of surgery for a distal radial fracture. The parameters of acceptable reduction associated with an improved outcome include restoration of radial length and minimization of the post- operative fracture gap and step. The mechanism used for fracture reduction is ligamentotaxis of the dorsal and volar capsules, which realigns the fracture with respect to the appropriate length, inclination and tilt. Our results did not show plating to be superior to external fixation in its ability to maintain proper reduction.

Higher rates of K-wire infection and hardware failure have been reported in patients treated with external fixation, and higher rates of tendon complications with internal fixation. ${ }^{23}$ Our patients undergoing external fixation had lower rates of deep infection and hardware failure than the patients in the study by Margaliot. ${ }^{23}$ We found eight pin-track infections, but most were minor and transient. One patient in the external fixation group and six in the plating group acquired deep wound infections. Using a Cox proportional hazard model, we determined that the only factor significantly related to patient cure rates was wound infection. Some studies have reported that the rate of major complications, such as redislocations requiring reoperation or complex regional pain syndrome, is higher in patients undergoing external fixation. ${ }^{24}$ Our external fixation group had somewhat better results than the patients in the study by Margaliot. ${ }^{23}$ Only three patients required further surgery for redislocation. By contrast, eight patients in the plating group required further surgery to treat wound infections and tendon-related complications.

The malunion or nonunion rates are important parameters for evaluating these two surgical methods and should be considered in the overall decision as to which method is used. In our study, only three cases in the internal fixation group and one case in the external fixation group had malunions requiring further surgery.

No psychosocial follow-up studies pertaining to IFDR treatment have been published, and our study is the first to take a long-term view (mean period of follow-up was 1 year). Furthermore, we used standardized questionnaires encompassing a wide range of psychosocial functioning domains. This method of testing health status, or HRQOL, is similar to the methodology used by McKee et al. $^{25}$ and Vitale et $a .^{26}$ Assessment of pain perception using an HRQOL questionnaire in our study yielded results similar to those of a study by McKee et al. ${ }^{25}$ In the comparison, we had expected to find differences, but the only difference we found in the TAAQOL scores between the internal fixation group and the external fixation group was a lower score in the "gross motor" category. There were no statistically significant differences in perceived competence and self-esteem in the other categories (Table 8). Our findings could be useful for deciding between external or internal procedures for treating IFDR. Patients who plan to undergo such a procedure should be informed that the procedure might involve a stressful and painful recovery. 
There were no statistically significant differences between the two groups with respect to recovery.

The role of this surgery in elderly patients requires further evaluation. The results of our observational study demonstrate higher complication rates in patients who underwent volar or dorsal locking plate treatment for IFDR. Furthermore, elderly patients who received greater anatomical reduction through volar or dorsal locking plate treatment did not necessarily show a better functional outcome. A large randomized, controlled trial is needed to clarify the differences in these methods and help guide treatment and counseling for elderly patients.

\section{Acknowledgements}

We thank Dr. Qin Wei for technical help and Dr. Bo Cao for assistance in collecting patients' data. This work was supported by National Natural Science Foundation of China Grant 81560350.

\section{Author contributions}

LC and AY planned and designed this study. CM wrote and translated this article. QD and HP performed the radiographic examinations. $\mathrm{XC}$ and $\mathrm{YK}$ gathered data from the patients and created the figures and tables. JY participated in the statistical analysis. All authors read and approved the final manuscript.

\section{Competing interests}

The authors declare no conflict of interest.

\section{References}

1 Xu GG, Chan SP, Puhaindran ME et al. Prospective randomised study of intra-articular fractures of the distal radius: comparison between external fixation and plate fixation. Ann Acad Med Singapore 2009; 38: 600-606.

2 Abramo A, Kopylov P, Geijer M et al. Open reduction and internal fixation compared to closed reduction and external fixation in distal radial fractures: a randomized study of 50 patients. Acta Orthop 2009; 80: 478-485.

3 den Dulk M, Marijnen CA, Putter $\mathrm{H}$ et al. Risk factors for adverse outcome in patients with rectal cancer treated with an abdominoperineal resection in the total mesorectal excision trial. Ann Surg 2007; 246: 83-90.

4 Arora R, Lutz M, Deml C et al. A prospective randomized trial comparing nonoperative treatment with volar locking plate fixation for displaced and unstable distal radial fractures in patients sixty-five years of age and older. J Bone Joint Surg Am 2011; 93: 2146-2153.

5 Chung KC, Petruska EA. Treatment of unstable distal radial fractures with the volar locking plating system. Surgical technique. J Bone Joint Surg Am 2007; 89 (Suppl 2)(Pt.2): 256-266.

6 Khanduja V, Ng L, Dannawi Z et al. Complications and functional outcome following fixation of complex, intra-articular fractures of the distal radius with the AO Pi-Plate. Acta Orthop Belg 2005; 71: 672-677.

7 Arora R, Lutz M, Hennerbichler A et al. Complications following internal fixation of unstable distal radius fracture with a palmar locking-plate. J Orthop Trauma 2007; 21: 316-322.
8 Lutz K, Yeoh KM, MacDermid JC et al. Complications associated with operative versus nonsurgical treatment of distal radius fractures in patients aged 65 years and older. J Hand Surg Am 2014; 39: 1280-1286.

9 Chung KC, Squitieri L, Kim HM. Comparative outcomes study using the volar locking plating system for distal radius fractures in both young adults and adults older than 60 years. J Hand Surg Am 2008; 33: 809-819.

10 Kapoor H, Agarwal A, Dhaon BK. Displaced intra-articular fractures of distal radius: a comparative evaluation of results following closed reduction, external fixation and open reduction with internal fixation. Injury 2000; 31: 75-79.

11 Estrella EP, Panti PL. Outcome of unstable distal radius fractures treated with open reduction and internal fixation versus external fixation. Hand Surg 2012; 17: 173-179.

12 Wei DH, Raizman NM, Bottino CJ et al. Unstable distal radial fractures treated with external fixation, a radial column plate, or a volar plate. A prospective randomized trial. J Bone Joint Surg Am 2009; 91: 1568-1577.

13 Gereli A, Nalbantoglu U, Kocaoglu B et al. Comparison of palmar locking plate and K-wire augmented external fixation for intra-articular and comminuted distal radius fractures. Acta orthopaedica et traumatologica turcica 2010; 44: 212-219.

14 Fracture and dislocation compendium. Orthopaedic Trauma Association Committee for Coding and Classification. J Orthop Trauma 1996; 10 Suppl 1:1-154.

15 Kumar S, Chopra RK, Sehrawat S et al. Comparison of treatment of unstable intra articular fractures of distal radius with locking plate versus non-locking plate fixation. J Clin Orthop Trauma 2014; 5: 74-78.

16 Hudak PL, Amadio PC, Bombardier C. Development of an upper extremity outcome measure: the DASH (disabilities of the arm, shoulder and hand) [corrected]. The Upper Extremity Collaborative Group (UECG). Am J Ind Med 1996; 29: 602-608.

17 Atroshi I, Gummesson C, Andersson B et al. The disabilities of the arm, shoulder and hand (DASH) outcome questionnaire: reliability and validity of the Swedish version evaluated in 176 patients. Acta Orthop Scand 2000; 71: 613-618.

18 Veerman JW, ten Brink LT, Straathof MA et al. Measuring children's selfconcept with a Dutch version of the "self-perception profile for children": factorial validity and invariance across a nonclinic and a clinic group. J Pers Assess 1996; 67: 142-154.

19 Theunissen NC, Vogels TG, Koopman HM et al. The proxy problem: child report versus parent report in health-related quality of life research. Qual Life Res 1998; 7: 387-397.

20 Egol K, Walsh M, Tejwani N et al. Bridging external fixation and supplementary Kirschner-wire fixation versus volar locked plating for unstable fractures of the distal radius: a randomised, prospective trial. J Bone Joint Surg Br 2008; 90: 1214-1221.

21 Marcheix PS, Dotzis A, Benko PE et al. Extension fractures of the distal radius in patients older than 50: a prospective randomized study comparing fixation using mixed pins or a palmar fixed-angle plate. J Hand Surg Eur Vol 2010; 35: 646-651.

22 Wilcke MK, Abbaszadegan H, Adolphson PY. Wrist function recovers more rapidly after volar locked plating than after external fixation but the outcomes are similar after 1 year. Acta Orthop 2011; 82: 76-81.

23 Margaliot Z, Haase SC, Kotsis SV et al. A meta-analysis of outcomes of external fixation versus plate osteosynthesis for unstable distal radius fractures. J Hand Surg Am 2005; 30: 1185-1199. 
24 Anderson JT, Lucas GL, Buhr BR. Complications of treating distal radius fractures with external fixation: a community experience. Iowa Orthop J 2004; 24: 53-59.

25 McKee MD, Yoo D, Schemitsch EH. Health status after Ilizarov reconstruction of post-traumatic lower-limb deformity. J Bone Joint Surg Br 1998; 80: 360-364.

26 Vitale MA, Choe JC, Sesko AM et al. The effect of limb length discrepancy on health-related quality of life: is the ' $2 \mathrm{~cm}$ rule' appropriate? J Pediatr Orthop B 2006; 15: 1-5. (i) This work is licensed under a Creative Commons Attribution 4.0 International License. The images or other third party material in this article are included in the article's Creative Commons license, unless indicated otherwise in the credit line; if the material is not included under the Creative Commons license, users will need to obtain permission from the license holder to reproduce the material. To view a copy of this license, visit http:/ / creativecommons.org/licenses/by/4.0/

(C) The Author(s) 2016 